\title{
Floquet Weyl Semimetal Induced by Off-Resonant Light
}

\author{
Rui Wang ${ }^{1}$, Baigeng Wang ${ }^{1}$, Rui Shen ${ }^{1}$, L. Sheng ${ }^{1}$, D. Y. Xing ${ }^{1}$ and Sergey Y. Savrasov ${ }^{1,2}$ \\ ${ }^{1}$ National Laboratory of Solid State Microstructures and School of Physics, Nanjing University, Nanjing 210093, China \\ ${ }^{2}$ Department of Physics, University of California, Davis, California 95616, USA
}

(Dated: September 10, 2013)

\begin{abstract}
We propose that a Floquet Weyl semimetal state can be induced in three-dimensional topological insulators, either nonmagnetic or magnetic, by the application of off-resonant light. The virtual photon processes play a critical role in renormalizing the Dirac mass and so resulting in a topological semimetal with vanishing gap at Weyl points. The present mechanism via off-resonant light is quite different from that via on-resonant light, the latter being recently suggested to give rise to a Floquet topological state in ordinary band insulators.
\end{abstract}

PACS numbers: 73.43.Nq, 73.20.At, 03.65.Vf

Introduction-Topological states of matter, such as two-dimensional (2D) quantum spin Hall insulators or 3D topological insulators (TIs) [1 8], have received a great interest in recent years. Searching for such states in solid state materials [8 10] has partly gained success; however, candidate materials for TIs are still very limited. Inspiringly, an intriguing method was put forward to realize topologically non-trivial phases in nonequilibrium by applying time-dependent perturbations to trivial phases [11 16]. Typical examples are the optically-activated anomalous Hall effect and spin Hall effect in $n$-doped paramagnetic semiconductors [14], and the so-called Floquet topological insulator (FTI) suggested by Lindner, Refael, and Galitski [16], whose quasienergy spectrum exhibits a single pair of helical edge states due to the on-resonant-light- induced band inversion. In the bulk FTI spectrum there is an avoided crossing separating the reshuffled valence band from the conduction band.

Recently, another type of topological phase termed the Weyl semimetal (WSM) [17] has attracted much attention. The WSM has no bulk gap but enjoys gapless nodes distributed in momentum space, and it possesses chiral surface states and Fermi arcs terminating at Weyl points with opposite chirality. Due to its remarkable electromagnetic properties such as the anomalous Hall effect [18, 19] and chiral magnetic effect [20], the WSM is regarded as a promising candidate for future applications in spintronics. Unfortunately, despite several proposals have been put forward in various systems [17, 19, 21], the WSM has not been realized experimentally yet. As a result, theoretical proposals on the WSM state that are feasible for experimental realization are still highly desirable.

In this Letter, we show that a non-equilibrium WSM state can be induced in 3D topological insulators by the application of off-resonant light, using the Floquet picture. In contrast to the on-resonant optical induction in Ref. [16], we focus on the off-resonant effect of light on band structures, and the underlying physics is outlined as follows. Considers a two-band system with bulk energy (a)

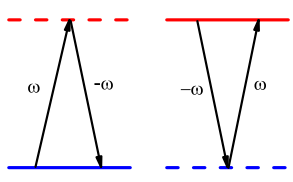

Virtual Photon Process

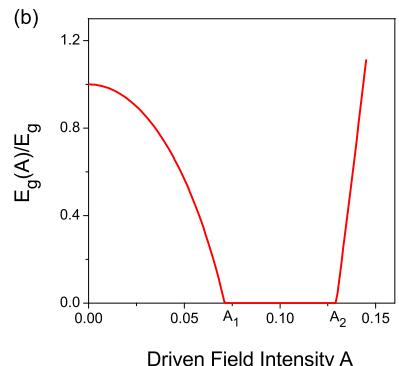

FIG. 1: (color online) (a)Schematic diagram for virtual photon processes. (b)Renormalized energy gap as a function of light field intensity. Here $A_{1}$ and $A_{2}$ are the thresholds (in unit of $\AA^{-1}$ ), at which a pair of Weyl points are generated and annihilated, respectively.

gap $E_{g}$ under the application of off-resonant light. For light with off-resonant frequency $\omega$, the real process of photon absorption/emission cannot occur because of limitation of energy conservation condition. However, the off-resonant light can affect the electron system via virtual photon processes, e.g., two second-order processes shown in Fig. 1(a), where electrons absorb and then emit a photon and electrons first emit and absorb a photon. Such virtual photon processes have at least two effects. First, they can break the time reversal symmetry (TRS) if the driven field is nonlinearly polarized, which is one of essential conditions to realize WSM. Second, they can generate a self-energy $\boldsymbol{\Sigma}$ that renormalizes the Dirac mass and so bulk energy gap $E_{g}(A)$ of the electron system. It will be shown that $E_{g}(A)$ first decreases with light field amplitude $A$ and vanishes at threshold $A_{1}$, as shown in Fig. 1(b). Once the light field amplitude is increased to another threshold $A_{2}$, the bulk gap reopens and $E_{g}(A)$ increases with further increasing $A$. For $A_{1}<A<A_{2}$, the bulk energy spectrum is gapless at Weyl points, and the system will be shown to be a Floquet WSM.

Floquet theory on topological insulator-We start from 
a modified Dirac Hamiltonian [22], which was used to describe magnetic 3D TI,

$$
H(\mathbf{k})=\sum_{i=1}^{3} a k_{i} \Gamma_{i}+M \Gamma_{4}+m \Gamma^{\prime} .
$$

The first two terms describe the nonmagnetic 3D TI, where $\Gamma_{i}(i=1, \ldots, 4)$ are the Dirac matrices that satisfy Clifford algebra in Euclidean space: $\left\{\Gamma_{i}, \Gamma_{j}\right\}=\delta_{i j}$. The Dirac mass is given by $M=M_{0}+b k^{2}$. It is well known that $M_{0} b<0$ guarantees a topologically nontrivial phase while $M_{0} b>0$ suggests a normal insulator (NI). Once $\Gamma_{1}, \ldots, \Gamma_{4}$ are specified, the other Dirac matrices can be constructed as $\Gamma_{0}=\mathbf{1}_{4 \times 4}, \Gamma_{5}=\prod_{i=0}^{4} \Gamma_{i}$, and $\Gamma_{i j}=$ $-\frac{i}{2}\left[\Gamma_{i}, \Gamma_{j}\right]$. The last term represents a time-reversalbreaking exchange field with $\Gamma^{\prime}$ satisfying $\left[\Gamma^{\prime}, \Gamma_{4}\right]=0$. A competition between $m \Gamma^{\prime}$ and $M \Gamma_{4}$ will enable the surface Dirac fermions to acquire mass, leading to a quantum anomalous Hall (QAH) effect. Since the choice of $\Gamma^{\prime}$ is flexible, we choose $\Gamma^{\prime}=\Gamma_{12}$ for the purpose of clarity.

An in-plane polarized electric field can be expressed by its vector potential $\mathbf{A}=\left(A_{x} \sin \omega t, A_{y} \sin (\omega t+\phi), 0\right)$ with $\omega$ as the frequency of the electric field and $\phi$ describing the polarization direction. For a spatially uniform electromagnetic field, the effect on the spin degree of freedom may be negligible while the effect on the orbital degree of freedom can be included through the substitution: $H(\mathbf{k}) \rightarrow H(\mathbf{k}+\mathbf{A}(t))$, yielding

$$
H(\mathbf{k}, t)=H(\mathbf{k})+\mathbf{V}(\mathbf{t}) \cdot \boldsymbol{\Gamma} .
$$

Here the second term is the time-dependent perturbation induced by the driven field with $\boldsymbol{\Gamma}=\left(\Gamma_{1}, \Gamma_{2}, \Gamma_{3}, \Gamma_{4}\right)$ and $\mathbf{V}(\mathbf{t})=\left(a A_{x} \sin \omega t, a A_{y} \sin (\omega t+\phi), 0, b A_{x}^{2} \sin ^{2} \omega t+\right.$ $\left.b A_{y}^{2} \sin ^{2}(\omega t+\phi)+2 b k_{x} A_{x} \sin \omega t+2 b k_{y} A_{y} \sin (\omega t+\phi)\right)$.

Due to the discrete time-translational invariance of $H(\mathbf{k}, t)$, the eigenvectors can be written as $\left|\psi_{\alpha}(t)\right\rangle=$ $e^{-i \epsilon_{\alpha} t}\left|\phi_{\alpha}(t)\right\rangle$, where $\left|\phi_{\alpha}(t)\right\rangle=\left|\phi_{\alpha}(t+T)\right\rangle$ is the Floquet state with $T=2 \pi / \omega$ and $\epsilon_{\alpha}$ is the quasi-energy restricted in the range of $-\omega / 2<\epsilon_{\alpha}<\omega / 2$. In the framework of the Floquet theory, the time-dependent Hamiltonian Eq. (2) can be mapped onto a time-independent Floquet operator $\mathbf{F}=\boldsymbol{\Omega} \otimes \mathbf{H}_{4 \times 4}$ in the Sambe space [23], with $\boldsymbol{\Omega}$ as an infinite-dimensional matrix in the Floquet band space and $\mathbf{H}_{4 \times 4}$ in the basis of $H(\mathbf{k})$ of the undriven system. The matrix element of the Floquet operator is given by

$$
\mathbf{F}_{m, n}(\mathbf{k})=H(\mathbf{k})+\boldsymbol{\Gamma} \cdot \mathbf{V}_{m, n}-n \omega \delta_{n m},
$$

where $\mathbf{V}_{m, n}=\frac{1}{T} \int_{-T / 2}^{T / 2} d t \mathbf{V}(\mathbf{t}) e^{-i(n-m) \omega t}$, and $m$ and $n$ denote the indices of Floquet bands. In principle, an exact diagonalization of the Floquet operator can give all the quasi-energy spectra. In order to obtain analytic results, we perform a perturbation theory, which holds for $a^{2} A_{x} A_{y} / M \omega \ll 1$ [24], to obtain the effective Floquet operator [1]]: $F_{\text {eff }}=F_{0,0}+\left[F_{-1,0}, F_{1,0}\right] / \omega$. Both terms
$F_{0,0}$ and $\left[F_{-1,0}, F_{1,0}\right] / \omega$ arise from the second-order virtual photon processes shown in Fig. 1(a), where a photon is first absorbed (released) and then released (absorbed). As will be demonstrated below, $\left[F_{-1,0}, F_{1,0}\right] / \omega$ breaks the TRS for a nonlinearly-polarized electric field, while $F_{0,0}$ differs from the undriven Hamiltonian $H(\mathbf{k})$ by a modification $\boldsymbol{\Sigma}$ that renormalizes the Dirac mass and generates Weyl points.

Using the perturbation theory, we obtain the effective Floquet operator around the $\Gamma$ point in momentum space as

$$
F_{\text {eff }}(\mathbf{k})=\sum_{i=1}^{3} a k_{i} \Gamma_{i}+\widetilde{M} \Gamma_{4}+\widetilde{m} \Gamma^{\prime},
$$

for the driven system, where $\widetilde{m}=m+a^{2}\left(A_{x} A_{y} / \omega\right) \sin \phi$ and $\widetilde{M}=\widetilde{M}_{0}+b k^{2}$ with $\widetilde{M}_{0}=M_{0}+b\left(A_{x}^{2}+A_{y}^{2}\right) / 2$. Comparing Eq. (4) with Eq. (11) for the undriven system, one finds two important differences between them. First, the renormalized exchange field is enhanced due to term $\left[F_{-1,0}, F_{1,0}\right] / \omega$. For a nonlinearly-polarized field $(\phi \neq 0)$, the TRS is broken even though there is no exchange field $(m=0)$. Second, the Dirac mass is dressed due to term $F_{0,0}$. Since the TI is chosen to be the initial state with $M_{0}>0$ and $b<0, \widetilde{M}_{0}$ is effectively reduced. Since $\widetilde{m}>m$ and $\widetilde{M}<M$, it is expected that the competition between $\widetilde{m} \Gamma^{\prime}$ and $\widetilde{M} \Gamma_{4}$ is favorable to emergence of the QAH phase and even WSM phase. If the field intensity is large enough to make $\widetilde{M}_{0}$ change its sign from positive to negative so that $\widetilde{M}_{0} b>0$, the system can be driven to be a topologically trivial insulator phase.

To be more explicit, we now represent the Dirac matrices as $\left(\Gamma_{1}, \Gamma_{2}, \Gamma_{3}, \Gamma_{4}\right)=\left(\tau^{x} s^{x}, \tau^{x} s^{y}, \tau^{x} s^{z},-\tau^{z} s^{0}\right)$, where $\mathbf{s}(\tau)$ is the Pauli matrix in spin (orbital) space. In this representation, Eq. (4) reads

$$
F_{\text {eff }}(\mathbf{k})=a \tau^{x}\left(s^{x} k_{x}+s^{y} k_{y}+s^{z} k_{z}\right)-\widetilde{M} \tau^{z} s^{0}+\widetilde{m} \tau^{0} s^{z} .
$$

In the absence of the last term, Eq. (5) is an isotropic version of the model in Ref. [8], which describes the 3D $\mathrm{TI} \mathrm{Bi}_{2} \mathrm{Se}_{3}$. To see the possibility of the topological phase transition, we first make a rotation in orbital space: $\tau^{x} \rightarrow$ $\tau^{z}$ and $\tau^{z} \rightarrow-\tau^{x}$, and then a canonical transformation: $\tau^{ \pm} \rightarrow s^{z} \tau^{ \pm}$and $s^{ \pm} \rightarrow s^{ \pm} \tau^{z}$. After $F_{\text {eff }}(\mathbf{k})$ in Eq. (5) is diagonalized in orbital space, we have

$$
F_{ \pm}(\mathbf{k})=a\left(s^{x} k_{x}+s^{y} k_{y}\right)+s^{z} \Delta_{ \pm}(\mathbf{k}),
$$

with $\Delta_{ \pm}(\mathbf{k})=\widetilde{m} \pm \sqrt{\widetilde{M}^{2}+a^{2} k_{z}^{2}}$. For a fixed $k_{z}$, Eq. (6) is a 2D Dirac Hamiltonian. It is well known that a sign change of the 2D Dirac mass signals a topological phase transition, characterized by a change of the first Chern number, which provides a method to distinguish different phases. From $\Delta_{-}\left(0,0, k_{z}\right)=0$, we have solution $k_{z}=$ $\pm k_{z c}$ with $a k_{z c}=\sqrt{\widetilde{m}^{2}-\widetilde{M}^{2}}$. For $\widetilde{m}^{2}>\widetilde{M}^{2}$, there are two real roots $\pm k_{z c}$ for $k_{z}$, and $\left(0,0, \pm k_{z c}\right)$ are just 

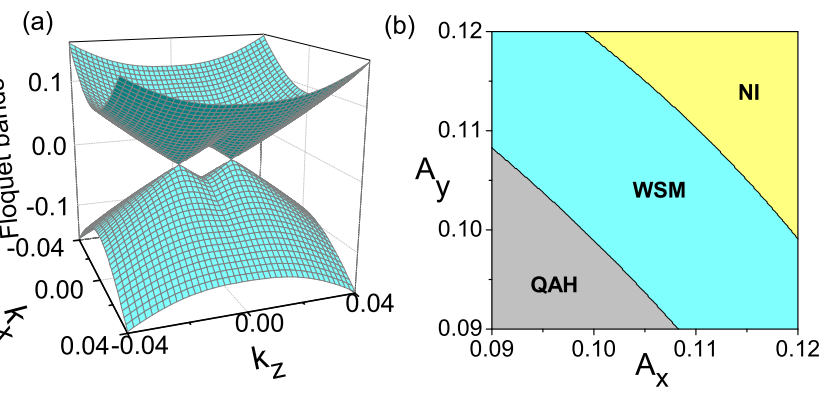

FIG. 2: (color online) (a) Calculated Floquet bands for a 3D TI subjected to a circularly polarized light with $A_{x}=$ $A_{y}=0.105 \AA^{-1}$, and (b) the corresponding topological phase diagram. The parameters are chosen as $M_{0}=0.28 \mathrm{eV}, a=$ $2.8 \mathrm{eV} \AA, b=-25.7 \mathrm{eV} \AA^{2}$, and $\omega=3 \mathrm{eV}$. (a)

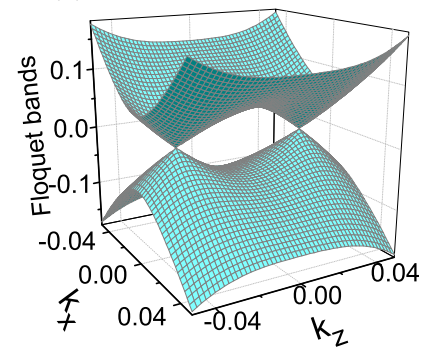

(b)

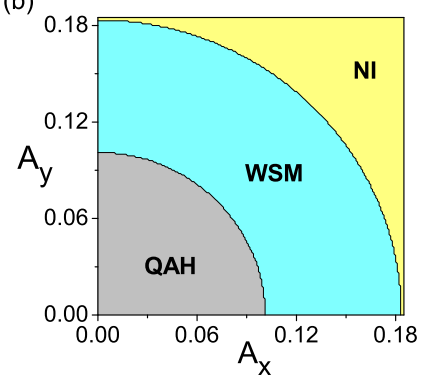

FIG. 3: (color online) (a) Calculated Floquet bands for a 3D magnetic TI subjected to a linearly polarized light with $A_{x}=A_{y}=0.1 \AA^{-1}$, and (b) the corresponding topological phase diagram. The exchange energy is chosen as $m=0.15$ $\mathrm{eV}$ and the other parameters are the same as in Fig. 2(a).

the two Weyl points in momentum space of the WSM. Further more, an expansion of Floquet operator $F_{-}$in Eq. (6) near the two gapless nodes $\pm k_{z c}$ yields

$$
F_{-}(\mathbf{k})=a \tau^{x} q_{x}-a \tau^{y} q_{y} \mp a \sqrt{\widetilde{m}^{2}-M_{0}^{2}} \tau^{z} q_{z},
$$

which is the same as the Hamiltonian defining the Weyl fermions with chirality $C= \pm 1$. Here $b k^{2}$ in the Dirac mass is temporarily neglected for clarity. On the other hand, for $\widetilde{m}^{2}<\widetilde{M}^{2}$, there exists no real root of $k_{z c}$ for $\Delta_{-}\left(0,0, k_{z}\right)=0$ and so the quasi-energy spectrum is gapped throughout the momentum space. In this case, either QAH or NI phase is possible, depending on whether $\widetilde{M}_{0} b<0$ or $\widetilde{M}_{0} b>0$. As a result, there is a topological phase transition at $\widetilde{m}^{2}=\widetilde{M}^{2}$ from the WSM phase to either QAH or NI phase.

From Eq. (5) we have numerically calculated the bulk quasi-energy dispersion in a single Floquet band for different polarizations. We first consider the case of $m=0$ and $\phi=\pi / 2$ (circularly polarized light). It is found that with increasing the off-resonant light intensity, the energy gap $E_{g}$ of the undriven TI becomes smaller and smaller, and vanishes at $k_{z c}=0$. With further increasing $A$, a pair of Weyl points at $\pm k_{z c}$ are separated from each other, as shown in Fig. 2(a). A continued increase of $A$ can make two Weyl points merge into a single Dirac point at $k_{z c}=0$, and then the gap reopens, resulting in a topologically trivial insulator. Figure 2(b) is the calculated phase diagram, where the WSM phase is in between the QAH and NI phases. Second, we consider the linearly-polarized case of $\phi=0$ with $m \neq 0$ taken. Similar topological phase transitions are obtained, as shown in Fig. 3. If $\phi=0$ and $m=0$, the system still has the TRS. In this case, there is no WSM phase in the phase diagram, and the TI phase is transited to the NI phase under the application of off-resonant light.

Lattice model-In what follows we study a more realistic diamond lattice model to support the above results. Its tight-binding Hamiltonian is given by

$$
H=t \sum_{\langle i j\rangle} c_{i}^{\dagger} c_{j}+i\left(8 \lambda_{S O} / a^{2}\right) \sum_{\langle\langle i j\rangle\rangle} c_{i}^{\dagger} \mathbf{s} \cdot\left(\mathbf{d}_{i j}^{1} \times \mathbf{d}_{i j}^{2}\right) c_{j} .
$$

This model was introduced to describe 3D TI [5]. Here we take $t_{1}=t+\delta t$ and $t_{2}=t_{3}=t_{4}=t$ with the distortion $\delta t$ along the (111) direction. For $\delta t>0$, the system lies in the strong topological insulator (STI) phase with $Z_{2}$ index as $\nu_{0}=1$, and $\delta t<0$ corresponds a weak topological insulator (WTI) phase with $\nu_{0}=0$.

First, we do not consider Zeeman splitting and only focus on the linearly polarized electric field $\mathbf{A}=$ $\left(A_{x} \sin \omega t, A_{y} \sin \omega t, 0\right)$. Using the procedure same as above, we obtain the Floquet operator in Sambe space as

$$
F_{m, n}(\mathbf{k})=\boldsymbol{\Gamma} \cdot \mathbf{D}_{q}(\mathbf{k})-n \omega \delta_{m n},
$$

where $m$ and $n$ denote different Floquet bands and $q=m-n$. The Dirac matrices in this case are represented as $\boldsymbol{\Gamma}=\left(\tau^{z} s^{x}, \tau^{z} s^{y}, \tau^{z} s^{z}, \tau^{x} s^{0}, \tau^{y} s^{0}\right)$, where $\tau$ and $s$ are the Pauli matrices associated with A-B sublattice and true spin subspace, respectively; and $\mathbf{D}_{q}(\mathbf{k})=$ $\left(d_{1 q}(\mathbf{k}), d_{2 q}(\mathbf{k}), d_{3 q}(\mathbf{k}), d_{4 q}(\mathbf{k}), d_{5 q}(\mathbf{k})\right)$, where $d_{i q}(\mathbf{k})$ are expressed via the $q$ th order Bessel functions, which can be obtained by first Fourier transforming Eq. (8) and then mapping it onto the Floquet operator in Sambe space. In the off-resonant region of $\omega>8 t$, we set $m=n=0$ in Eq. (9) to obtain $F_{0,0}$, which is the most relevant contribution from all the virtual photon processes.

In Eq. (9) for $q=0$, both the TRS and the inversion symmetry, which can be represented as $\hat{\Theta}=i s^{y} K$ and $\hat{P}=\tau^{x} s^{0}$, respectively [7], remain unchanged. Therefore, the $Z_{2}$ index of the driven Hamiltonian can be conveniently calculated, which is modulated by the offresonant light. Figure 4(a) shows the phase diagram, in which there is a topological phase transition between the STI and WTI phases.

Next, we take the Zeeman splitting into account with term $m \tau^{0} s^{z}$ included in Eq. (91). The eigenvalues are obtained as $E_{ \pm}^{2}=d_{1}^{2}+d_{2}^{2}+\left(\sqrt{d_{3}^{2}+d_{4}^{2}+d_{5}^{2}} \pm m\right)^{2}$. Note 

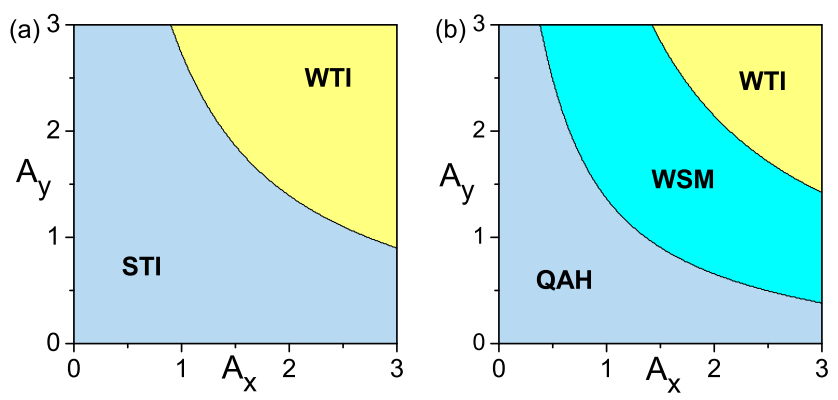

FIG. 4: (color online) Calculated topological phase diagrams of the diamond lattice model driven by a linearly polarized light $(\phi=0)$ with $m=0$ (a) and $m=0.2 \mathrm{eV}$ (b). The parameters are taken as $t_{2,3,4}=1, t_{1}=1.4$, and $\lambda_{S O}=0.05$.

that there are gapless nodes only for $E_{-}$, which requires $d_{1}=d_{2}=0$ and $m=\sqrt{d_{3}^{2}+d_{4}^{2}+d_{5}^{2}}$. There are three equations that determine three variables $k_{x}, k_{y}$, and $k_{z}$. The solutions for them are exactly the Weyl nodes in momentum space. To make this argument more convincing, we expand the Floquet operator around point $X^{z}=(0,0,2 \pi)$ and then make the canonical transformation $s^{ \pm} \rightarrow \tau^{z} s^{ \pm}$and $\tau^{ \pm} \rightarrow \tau^{ \pm} s^{z}$ to obtain the effective Hamiltonian as

$$
H_{e f f}=\lambda_{S O}\left(s^{x} q_{x}-s^{y} q_{y}\right)+s^{z} \Delta^{ \pm}(\mathbf{q} ; \mathbf{A}) .
$$

Here the effect of the light field $\mathbf{A}$ has been absorbed into the Dirac mass: $\Delta^{ \pm}(\mathbf{q} ; \mathbf{A})=m \pm\left[M_{z}^{2}+h_{a b} q_{a} q_{b}\right]^{1 / 2}$ with $M_{z}=\sum_{i=1}^{4} \operatorname{sgn}\left(\mathbf{e}_{\mathbf{i}} \cdot \hat{\mathbf{z}}\right)$, where $\mathbf{e}_{\mathbf{i}}$ is the nearest bond vector of the diamond lattice and $h_{a b}$ is a tensor describing the anisotropy that is determined by field parameters $A_{x}$ and $A_{y}$. Equation (10) is of the same form as Eq. (6), so that a similar analysis can be made here. The numerical results searching for different phases are shown in Fig. 4(b), in which the WSM phase is in between the QAH and magnetic WTI phases.

Experimental realization and conclusion-First, in order to take advantage of the off-resonant process, the frequency of the electric field should be of the order of $10^{3} \mathrm{THz}$. Therefore, an ultraviolet light is qualified. Second, the field intensity has to cross the topological phase transition line as shown in Fig. 3, i.e., $A_{0}>0.1 \AA^{-1}$. In terms of the electric field amplitude $E_{0}$, it should be of the order of $0.1 V \AA^{-1}$. It needs to be stressed that the amplitude threshold will be greatly reduced for a TI material with a smaller $M_{0}$ and a larger $|b|$. For example, $E_{0}$ is reduced to $10^{-4} V \AA^{-1}$ for $M_{0} \sim 0.01 \mathrm{eV}$ and $b \sim-100 e V \AA^{2}$.

In conclusion, we have shown that the Floquet WSM phase can be induced in 3D TIs by the use of the offresonant light. The virtual photon absorption/emission processes play a key role in renormalizing the Dirac mass, in closing the bulk gap, as well as in breaking the TRS, resulting in a nontrivial WSM phase. From both the continuous and lattice models of the Floquet theory, very similar phase diagrams have been obtained. With increasing the light intensity, the QAH phase first transits to the WSM phase and then to the NI or magnetic WTI phase. According to recent experiments such as the observation of the QAH effect in magnetic TIs 25] and the realization of Floquet topological insulators in a photonic system [26], the present proposal of the Floquet WSM state can be realized in experiments. In addition, we wish to point out that the generalized Dirac Hamiltonian, Eq. (11), can describe not only the 3D TIs, but also the other systems such as the polyacetylene, $p$-wave pairing superconductor, and ${ }^{3} \mathrm{He}-\mathrm{A}$ and ${ }^{3} \mathrm{He}-\mathrm{B}$ phases 22 . Therefore, it is expected that the light field may also induce topological phase transitions in those systems.

This work is supported by the State Key Program for Basic Research of China under Grant No. 2011CB922103, and by the National Natural Science Foundation of China under Grants No. 60825402, No. 11023002, and No. 91021003.

[1] C. L. Kane and E. J. Mele, Phys. Rev. Lett. 95, 226801 (2005).

[2] C. L. Kane and E. J. Mele, Phys. Rev. Lett. 95, 146802 (2005).

[3] B. A. Bernevig and S. C. Zhang, Phys. Rev. Lett. 96, 106802 (2006).

[4] C. J. Wu, B. A. Bernevig, and S. C. Zhang, Phys. Rev. Lett. 96, 106401 (2006).

[5] L. Fu, C. L. Kane, and E. J. Mele, Phys. Rev. Lett. 98, 106803 (2007).

[6] J. E. Moore and L. Balents, Phys. Rev. B 75, 121306 (2007).

[7] L. Fu and C. L. Kane, Phys. Rev. B 76, 045302 (2007).

[8] H. Zhang, C.-X. Liu, X.-L. Qi, X. Dai, Z. Fang, and S.-C. Zhang, Nature Phys. 5, 438 (2009).

[9] B. A. Bernevig, T. L. Hughes, and S-C. Zhang, Science 314, 1757 (2006).

[10] D. Hsieh, D. Qian, L.Wray, Y. Xia, Y. S. Hor, R. J. Cava and M. Z. Hasan, Nature 452, 970 (2008).

[11] T. Kitagawa, T. Oka, A. Brataas, L. Fu, and E. Demler, Phys. Rev. B 84, 235108 (2011).

[12] Z. H. Gu, H. A. Fertig, D. P. Arovas, and A. Auerbach, Phys. Rev. Lett. 107, 216601 (2011).

[13] Eric Suárez Morell and Luis E. F. Foa Torres, Phys. Rev. B 86, 125449 (2012).

[14] W. Yao, A. H. MacDonald, and Q. Niu, Phys. Rev. Lett. 99, 047401 (2011).

[15] T. Kitagawa, E. Berg, M. Rudner, and E. Demler, Phys. Rev. B 82, 235114 (2010).

[16] N. H. Lindner, G. Refael and V. Galitski, Nature Phys. 7, 490 (2011).

[17] X. G. Wan, A. M. Turner, A. Vishwanath, and S. Y. Savrasov, Phys. Rev. B 83, 205101 (2011).

[18] K. Y. Yang, Y. M. Lu, and Y. Ran, Phys. Rev. B 84, 075129 (2011). 
[19] A. A. Burkov and L. Balents, Phys. Rev. Lett. 107, 127205 (2011).

[20] K. Fukushima, D. E. Kharzeev, and H. J. Warringa, Phys. Rev. D 78, 074033 (2008).

[21] G. Y. Cho, arXiv:1110.1939.

[22] S. Q. Shen, Topological Isulators-Dirac Equation in Condensed Matters (Springer Series in solid-state sciences, 2012).

[23] H. Sambe, Phys. Rev. A 7, 2203 (1973).

[24] This condition is not nessesary in the lattice model, because the vector potential enters into $F_{m, n}$ through $(m-n)-$ th order Bessel functions, which decay oscillatorily.

[25] C.-Z. Chang, J. Zhang, X. Feng, J. Shen, Z. Zhang, M. Guo, K. Li, Y. Ou, P. Wei, L.-L. Wang, Z.-Q. Ji, Y. Feng, S. Ji, X. Chen, J. Jia, X. Dai, Z. Fang, S.-C. Zhang, K. He, Y. Wang, L. Lu, X.-C. Ma, Q.-K. Xue, Science 340, 167 (2013).

[26] M. C. Rechtsman, J. M. Zeuner, Y. Plotnik, Y. Lumer, D. Podolsky, F. Dreisow, S. Nolte, M. Segev \& Al. Szameit, Nature 496, 196 (2013). 